\title{
PRMT1 Promoted HCC Growth and Metastasis In Vitro and In Vivo via Activating the STAT3 Signalling Pathway
}

\author{
Xiu-Ping Zhang ${ }^{\mathrm{a}}$ Ya-Bo Jiang ${ }^{\mathrm{a}}$ Cheng-Qian Zhonga,c Ning Ma ${ }^{\mathrm{b}}$ \\ Er-Bin Zhang ${ }^{b}$ Fan Zhang ${ }^{d}$ Jing-Jing Lib Yue-Zhen Deng ${ }^{b} \quad K^{b}$ ang Wang ${ }^{a}$ \\ Dong Xie $^{\mathrm{b}}$ Shu-Qun Cheng ${ }^{\mathrm{a}}$
}

\begin{abstract}
aDepartment of Hepatic Surgery VI, Eastern Hepatobiliary Surgery Hospital, Second Military Medical University, Shanghai, 'Laboratory of Molecular Oncology, Institute for Nutritional Sciences, Shanghai Institutes for Biological Sciences, University of Chinese Academy of Sciences, Shanghai, 'LongYan First Hospital, Affiliated to Fujian Medical University, FuJian, China, dDepartment of Hepatic Surgery, BinZhou Medical University Hospital, BinZhou, China
\end{abstract}

\section{Key Words}

Protein arginine methyltransferase 1 - Hepatocellular carcinoma • STAT3 signalling pathway • Cryptotanshinone

\begin{abstract}
Background/Aims: Although it has been widely accepted that protein arginine methyltransferase 1 (PRMT1) is a cancer-promoting gene in various cancers, the mechanism of PRMT1 in hepatocellular carcinoma (HCC) requires more exploration. This study aimed to investigate the role of PRMT1 in HCC growth and metastasis. Methods: We compared PRMT1 expression and clinicopathological characteristics using paired HCC and adjacent noncancerous liver tissues from 210 patients and immunohistochemistry analyses. Cell proliferation, colony formation and migration were determined in HCC cell lines with PRMT1 overexpression or downregulation through MTT, crystal violet and Boyden chamber assays. Tumour growth was monitored in a xenograft model, and intrahepatic metastasis models were established. Results: PRMT1 expression was greatly increased in clinical HCC samples and strongly associated with poor prognosis and recurrence; PRMT1 expression was also positively correlated with microvascular invasion $(P=0.024)$, tumour differentiation $(P=0.014)$, tumour size $(P=0.002)$, and portal vein tumour thrombus $(P V T T)(P=0.028)$. Cell proliferation, colony formation and migration in vitro were enhanced by PRMT1 upregulation and decreased by PRMT1 downregulation in HCC cell lines. Moreover, low PRMT1 expression resulted in slow tumour growth and decreased tumour weight in vivo, as well as tumour metastasis. These phenotypes were associated with STAT3 signalling pathway activation. Cryptotanshinone, a STAT3 inhibitor, inhibited STAT3 phosphorylation and reversed the HCC phenotype of PRMT1 expression. Conclusions: We revealed a significant role for PRMT1 in HCC progression and
\end{abstract}

X.-P. Zhang, Y.-B. Jiang and C.-Q. Zhong contributed equally to this work.

\begin{tabular}{ll}
\hline Shu-Qun Cheng, MD & Eastern Hepatobiliary Surgery Hospital, Second Military Medical University \\
& 225 Changhai Road, Shanghai 200433 (China) \\
& E-Mail chengshuqun@aliyun.com
\end{tabular}




\section{Cellular Physiology Cell Physiol Biochem 2018;47:1643-1654 \\ \begin{tabular}{l|l} 
DOI: 10.1159/000490983 & and Biochemistry \\
Published online: June 27, 2018 & $\begin{array}{l}\text { 2018 The Author(s). Published by S. Karger AG, Basel } \\
\text { www.karger.com/cpb }\end{array}$
\end{tabular} \\ Zhang et al.: PRMT1 Promoted HCC Growth and Metastasis}

metastasis in vitro and in vivo via STAT3 signalling pathway activation. PRMT1 may be a potential novel prognostic biomarker and new therapeutic target for HCC.

(C) 2018 The Author(s)

Published by S. Karger AG, Basel

\section{Introduction}

Hepatocellular carcinoma (HCC) is one of the most common malignant tumours with high morbidity and mortality [1]. Therapies for HCC have continued to evolve over the last decade; HCC patients can be treated using surgical resection, transarterial chemoembolization (TACE), radiotherapy and sorafenib. However, HCC patients cannot be totally cured due to prognosis difficulty, recurrence and early blood vessel invasion [2-5]. These problems cannot be solved because fundamental studies have not fully clarified the molecular mechanisms of HCC [6-8]. Therefore, it is important to fully understand HCC development and find new molecular targets for HCC treatment.

Protein arginine methyltransferase (PRMT) is a methyltransferase that can catalyse protein arginine methylation from S-adenosylmethionine (SAM) to arginine residues, which are involved in many cellular processes, such as signal transduction. PRMTs can be divided into four families: type I (PRMT1, PRMT3, PRMT4/CARM1, PRMT6 and PRMT8), type II (PRMT5, PRMT7 and PRMT10), and type IV (PRMT2) enzymes [9]. Currently, due to the close relationship between protein arginine methylation and various types of tumours, an increasing number of researchers have begun to study the relationship between PRMTs and cancer, especially PRMT1. PRMT1 regulates tumour growth and metastasis in human melanoma via targeting ALCAM [10]. In triple-negative breast cancer, PRMT1 is critical for epidermal growth factor receptor (EGFR) activity, and inhibiting PRMT1 may be an effective therapeutic strategy for overcoming intrinsic cetuximab resistance in triplenegative breast cancer cells [11]. Similarly, in oesophageal squamous cell carcinoma, PRMT1 activates Hedgehog signalling and upregulates the expression of target genes downstream of Hedgehog signalling to promote the growth and migration of esophageal squamous cell carcinoma (ESCC) cells [12]. One study preliminarily explored the molecular mechanisms of PRMT1 in HCC in vitro [13], but the clinicopathological significance of PRMT1 and HCC patient prognosis and the underlying mechanisms of PRMT1 in the promotion of HCC growth and metastasis in vitro and in vivo have not been examined.

Our study focused on the importance of PRMT1 in the progression of HCC. We found that HCC patients with high PRMT1 expression have a worse prognosis; in addition, we assessed the function of PRMT1 in HCC in vitro and in vivo. Finally, we determined that PRMT1 could accelerate HCC progression through increasing STAT3 phosphorylation.

\section{Materials and Methods}

\section{Patients and specimens}

HCC tissues and paired normal tissues were collected from 210 patients at Eastern Hepatobiliary Surgery Hospital (EHBH), Second Military Medical University, Shanghai, China. All tissues were frozen immediately in liquid nitrogen after surgical excision and stored at $-80{ }^{\circ} \mathrm{C}$. Informed written consent was obtained from all patients, and our study was approved by the Ethics Committee of EHBH and the Institutional Review Board of the Institute for Nutritional Sciences, Chinese Academy of Sciences.

\section{Immunohistochemistry}

Clinical HCC tissues and paired non-cancerous tissues were fixed in formalin, embedded in paraffin, and cut into $5-\mu \mathrm{m}$-thick consecutive sections. After deparaffinization and antigen recovery (in a sodium citrate solution, $\left.\mathrm{pH} 6.0,20 \mathrm{~min}, 98^{\circ} \mathrm{C}\right)$, the sections were washed thrice with $0.01 \mathrm{~mol} / \mathrm{l} \mathrm{PBS}(8 \mathrm{mmol} / \mathrm{l}$ $\mathrm{Na} 2 \mathrm{HPO} 4,2 \mathrm{mmol} / \mathrm{l} \mathrm{NaH2PO4}$, and $150 \mathrm{mmol} / \mathrm{l} \mathrm{NaCl}$ ) for 5 min each time, blocked for $1 \mathrm{~h}$ in $0.01 \mathrm{~mol} / \mathrm{l}$ PBS containing 0.3\% Triton X-100 and 5\% BSA, and incubated with an anti-PRMT1 (1:200) antibody (Cell Signaling Technology) at $4{ }^{\circ} \mathrm{C}$ overnight. After brief washes with $0.01 \mathrm{~mol} / \mathrm{l} \mathrm{PBS}$, the sections were incubated 


\section{Cellular Physiology Cell Physiol Biochem 2018;47:1643-1654

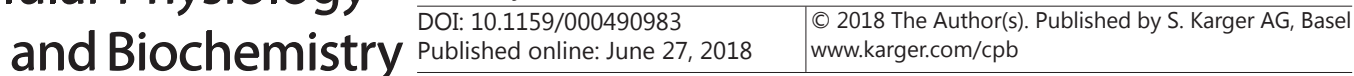 \\ Zhang et al.: PRMT1 Promoted HCC Growth and Metastasis}

with $0.01 \mathrm{~mol} / \mathrm{l}$ PBS containing a horseradish peroxidase-conjugated anti-rabbit IgG antibody (1:500) for 2 h, followed by development with $0.003 \% \mathrm{H} 202$ and $0.03 \% 3$, 30-diaminobenzidine in $0.05 \mathrm{~mol} / \mathrm{l}$ Tris- $\mathrm{HCl}$ (pH 7.6). Immunohistochemistry for each sample was repeated thrice.

\section{Cell lines and cell culture}

The normal liver cell line (LO2) and HCC cell lines (7721, HepG2, BEL-7404 and YY-8100) used in this study were purchased from the American Type Culture Collection (Manassas, VA, USA) and cultured in Dulbecco's modified Eagle's medium (DMEM) (Gibco) with 10\% foetal bovine serum and 1\% antibiotics (10 $\mathrm{U} / \mathrm{ml}$ penicillin and $10 \mathrm{U} / \mathrm{ml}$ streptomycin) in a sterile incubator with a humidified atmosphere containing $5 \% \mathrm{CO} 2$ at $37^{\circ} \mathrm{C}$.

\section{Establishment of PRMT1 overexpression cells}

The full-length cDNA encoding human PRMT1 was obtained from human whole blood by reverse transcription polymerase chain reaction (PCR). The human PRMT1 gene primer pair was designed using PRIMER5 software and is shown in Table 1. PRMT1 cDNA was cloned into a p23 vector (Takara Bio, Shiga, Japan) according to the manufacturer's instructions. Lentiviral supernatants were produced using the Lenti-X HTX packaging system (Clontech Laboratories, Mountain View, CA) and used for transducing the HCC cell lines Bel-7404 and YY-8103. For negative controls, we transduced the HCC cell lines with supernatants from empty vector cells. Fluorescence-activated cell sorting was performed twice to select stable clones. Over-expressed PRMT1 with a Flag tag was detected in cell lines with an anti-Flag antibody (Sigma).

\section{Establishment of PRMT1 knockdown cells}

Lentiviral short hairpin RNA (shRNA) transduction particles targeting PRMT1 and a negative control vector were purchased from Sigma-Aldrich (St. Louis, MO) and transduced into the HCC cell lines Bel-7404 and YY-8103 according to the manufacturer's instructions. Puromycin $(2.5 \mu \mathrm{g} / \mathrm{mL})$ was used to select stable clones. The shRNA sequences are shown in Table 1.

\section{RT-PCR analysis}

Total RNA was extracted from tissues and cells by using TRIzol reagent (Invitrogen). Then, the isolated RNA and a reverse transcription kit (Promega, Madison, WI) were used for cDNA synthesis according to the manufacturer's instructions. Real-time PCR (RT-PCR) experiments were performed with LightCycler DNA Master SYBR Green I mix (Roche Applied Science, Penzberg, Germany). The human PRMT1 gene primer pair was designed using PRIMER5 software and is shown in Table 1. The threshold cycle number (CT) was determined for both the PRMT1 gene and the control gene (GAPDH) with iCycler software. For each reaction, the mean CT of three reactions was calculated. The $\Delta \mathrm{CT}$ or the CT of the housekeeping gene (GAPDH) subtracted from the CT of the target gene (PRMT1) was plotted for each sample.

\section{Western blotting}

Tissue and cell protein was extracted by using tissue or cell lysis. The proteins were mixed with loading buffer (Bio-Rad, Hercules, CA) and boiled at $95^{\circ} \mathrm{C}$ for $5 \mathrm{~min}$. Then, 10\% SDS-PAGE was used to separate the total cell lysates, and the proteins were transferred onto polyvinylidene difluoride membranes (Millipore, Bedford, MA). The PVDF membranes were blocked with 5\% BSA for $1 \mathrm{~h}$ at room temperature and incubated overnight with primary antibodies in 1\% BSA. Then, the membranes were incubated with secondary antibody at a dilution of 1:5000 for $1 \mathrm{~h}$ at room temperature. Antibodies against PRMT1, Stat3 and p-Stat3 were purchased from Cell Signaling Technology. Antibodies against GAPDH were purchased from Sigma. An antibody against Flag was purchased from Sigma.

\section{MTT assay}

Three replicates of equal amounts of PRMT1 overexpression and sh-PRMT1 BEL7404 and YY-8103 cells $\left(1 \times 10^{3} /\right.$ well $)$ were seeded in 96-well plates for various durations; $5 \mathrm{mg} / \mathrm{mL} \quad 3-(4, \quad 5$-dimethylthiazol- 2-yl)-2,

Table 1. Primer sequence

\begin{tabular}{lcc}
\hline Gene & Forward & Reverse \\
\hline PRMT1 & 5'-GAGTTCACCCGATGCCACAAG-3' $^{\prime}$ & 5'-TCCGGTAGTCGGTGGAACAAG-3' $^{\prime}$ \\
& 5'-CGGCCGGCAGTACAAGACTAC $^{\prime}$ 5'-AATTCAAAAACGGCAGTACAAAGA \\
PRMT1-sh1 & AACTCGAGTTGTAGTCTTTGTACTGC & CTACAACTCGAGTTGAGTCTTTGTACTG \\
& CGGTTTTTG-3' & CCGG-3' \\
& 5'-CCGGGTGTTCCAGTATCTCTGAT & 5'-AATTCAAAAAGTGTTCCAGTATCTCT \\
PRMT1-sh2 & TACTCGAGTAATCAGAGATACTGGA & GATTACTCGAGTAATCAGAGATACTGGA \\
& ACACTTTTTG-3' & ACAC-3' \\
Myc & CAGCGACTCTGAAGAAGAGCA & TTGTGCTGGTGAGTGGAGAC \\
IL-6 & ACTCACCTCTTCAGAACGATTG & CCATCTTTGGAAGGTTCAGGTTG \\
VEGF & GAGGAGCAGTTACGGTCTTG & TCCTTTCCTTGCTGACACTTGT \\
GAPDH & 5'-ATGACCCCTTCATTGACCTCA-3' & 5'-GAGATGATGACCCTTTTGGCT-3' \\
\hline
\end{tabular}




\section{Cellular Physiology Cell Physiol Biochem 2018;47:1643-1654 and Biochemistry Published onl00490983 June 27, $2018 \quad \begin{aligned} & \text { DO 2018 The Author(s). Published by S. Karger AG, Basel } \\ & \text { www.karger.com/cpb }\end{aligned}$ \\ Zhang et al.: PRMT1 Promoted HCC Growth and Metastasis}

5-diphenyltetrazolium bromide (MTT, $20 \mu \mathrm{L} /$ well) was added to each well and incubated for $4 \mathrm{~h}$. Then, $200 \mu \mathrm{L}$ dimethyl sulfoxide (DMSO; Sangon Biotech, Shanghai, China) was added to the treated cells, and the optical density (OD) was detected at $490 \mathrm{~nm}$ using a microplate reader.

\section{Crystal violet assay}

The same number of control and experimental cells were seeded at a density of 3000 cells/well in 6-well plates and cultured in medium supplemented with 10\% FBS. The medium was changed every other day. After 10 days of culture under standard conditions, the medium was removed, and the cells were stained with $1 \mathrm{~mL} 0.5 \%$ crystal violet solution in $20 \%$ methanol. After staining for $10 \mathrm{~min}$, the fixed cells were washed with phosphate-buffered saline (PBS) and photographed. Then, $1 \mathrm{~mL}$ glacial acetic acid was added to the cells, and the optical density (OD) was detected at $570 \mathrm{~nm}$ by using a microplate reader.

\section{Boyden chamber assay}

Cells $\left(2 \times 10^{5}\right)$ suspended in $0.05 \mathrm{ml}$ medium containing $1 \%$ FBS were placed in the upper chamber, and the lower chamber was loaded with 0.152 ml medium containing $10 \%$ FBS. Six hours later, the cells that migrated to the lower surface of the filters were detected with traditional H\&E staining. The experiments were repeated thrice.

\section{Tumourigenesis in vivo}

Suspended cells $\left(2.5 \times 10^{6}\right)$ were injected into 5 -week-old male nude mice that were cared for in accordance with AAALAC criteria. Each animal was injected subcutaneously at two sites in their flanks. Tumour volume $(\mathrm{cm} 3)$ was measured every 5 th day, and tumour weight was measured at the end of the experiment.

\section{In vivo metastasis assay}

YY-8103 cells overexpressing luciferase were established by stable transfection. Luciferase activity was determined with the luciferin (Xenogen) in vivo imaging system (Xenogen). YY-8103/con cells overexpressing luciferase and YY-8103/sh-PRMT1 cells overexpressing luciferase $\left(2.5 \times 10^{6}\right.$ cells in 50 $\mu \mathrm{l}$ Matrigel) were injected into the left livers of the nude mice. The metastatic lesions were monitored. Before the mice were anaesthetized with isoflurane, luciferin $(150 \mathrm{mg} / \mathrm{kg}$ intraperitoneally) was injected into the nude mice 5 min before imaging. The nude mice were placed into the light-tight chamber of a CCD camera system (Xenogen), and the photons emitted from the luciferase-expressing cells within the animal were quantified for 1 min using the Living Image software program (Xenogen) as an overlay on Igor (Wavemetrics).

\section{Luciferase reporter assay}

BEL-7404 and YY-8103 cells were seeded in 24-well plates at an appropriate density and incubated for $24 \mathrm{~h}$. We used transient PRMT1-overexpressing cells for the STAT3 reporter assay. A total of $0.05 \mu \mathrm{g}$ STAT3 reporter plasmid (Institute of Health Sciences, Shanghai Institutes for the Biological Sciences, Chinese Academy of Sciences, Shanghai, China) and $0.02 \mu \mathrm{g}$ Renilla luciferase STAT3 as an internal control were cotransfected by using Lipofectamine 2000. Cell lysates were prepared $8 \mathrm{~h}$ after treatment, and the reporter activities were detected with a dual-luciferase reporter assay system (Promega, Madison, WI, USA).

\section{Statistical analysis}

Data from three independent experiments are expressed as the mean \pm standard deviation (SD). All statistical analyses were performed with SPSS statistics v21.0 (IBM Corp., Armonk, NY, USA). The differences between the experimental group and the control group were analysed by Student's t-test. P $<0.05$ was considered statistically significant.

\section{Results}

PRMT1 expression in HCC tissues and the clinicopathological significance of PRMT1 in HCC

To determine the expression level of PRMT1 in HCC tissues, we first used RT-PCR to compare the mRNA expression levels of PRMT1 in 35 paired HCC samples and matched KARGER 
normal tissues. The PRMT1 mRNA level was increased in HCC tissues (Fig. 1A). PRMT1 mRNA upregulation was detected in $85.7 \%(30 / 35)$ of HCC tissues $(\mathrm{P}<0.01)$ (Fig. 1A). Moreover, we randomly selected 10 pairs of HCC tissues and adjacent normal tissues and measured the protein expression of PRMT1 by Western blot. Increased PRMT1 protein levels were all detected in all 10 pairs of the HCC and normal tissues (Fig. 1B). Then, to further confirm the expression of PRMT1, we examined PRMT1 expression in an HCC tissue microarray by using immunohistochemical (IHC) staining. Consistent with the RT-PCR and Western blot results, PRMT1 expression was higher in the HCC tissues than in the normal tissues $(\mathrm{P}<0.001)$ (Fig. $1 \mathrm{C}$ and 1D). Moreover, we studied the relationship between the prognosis of HCC patients and the expression of PRMT1. We found that high PRMT1 expression levels were associated with poor overall survival and recurrence-free survival in 210 HCC patients (Fig. 1E and 1F). These results showed that PRMT1 was upregulated in HCC tissues and that PRMT1 overexpression affected the prognosis of HCC patients.

The staining intensity of PRMT1 expression was scored automatically with a Vectra2 system (Perkin Elmer, USA). According to the intensity score, the HCC patients were divided into two
Table 2. Correlation between PRMT1 expression and clinicopathological characteristics

\begin{tabular}{|c|c|c|c|c|}
\hline \multirow{2}{*}{ Variables } & \multirow{2}{*}{ Num. } & \multicolumn{2}{|c|}{ PRMT1, $105 \%$} & \multirow{2}{*}{ P-value } \\
\hline & & Low expression & High expression & \\
\hline Sex & & & & 0.158 \\
\hline Male & 190 & $92(87.62 \%)$ & $98(93.33 \%)$ & \\
\hline Female & 20 & $13(12.38 \%)$ & $7(6.67 \%)$ & \\
\hline Age, years & & & & 0.489 \\
\hline$<50$ & 111 & $58(55.24 \%)$ & $53(50.48 \%)$ & \\
\hline$\geq 50$ & 99 & $47(44.76 \%)$ & $52(49.52 \%)$ & \\
\hline AFP, $\mu \mathrm{g} / \mathrm{L}$ & & & & 0.23 \\
\hline$<20$ & 64 & $36(34.29 \%)$ & $28(26.67 \%)$ & \\
\hline$\geq 20$ & 146 & $69(65.71 \%)$ & $77(73.33 \%)$ & \\
\hline HBsAg & & & & 1 \\
\hline Negative & 16 & $8(7.62 \%)$ & $8(7.62 \%)$ & \\
\hline Positive & 194 & $97(92.38 \%)$ & $97(92.38 \%)$ & \\
\hline Ascites & & & & 0.603 \\
\hline Negative & 194 & $96(91.43 \%)$ & $98(93.33 \%)$ & \\
\hline Positive & 16 & $9(8.57 \%)$ & $7(6.67 \%)$ & \\
\hline PVTT & & & & 0.028 \\
\hline Negative & 181 & $96(91.43 \%)$ & $85(80.95 \%)$ & \\
\hline Positive & 29 & $9(8.57 \%)$ & $20(19.05 \%)$ & \\
\hline Tumour size, $\mathrm{cm}$ & & & & 0.002 \\
\hline$<5$ & 67 & $44(41.9 \%)$ & $23(21.9 \%)$ & \\
\hline$\geq 5$ & 153 & $61(58.10 \%)$ & $82(78.10 \%)$ & \\
\hline Small lesions & & & & $<0.001$ \\
\hline Negative & 137 & $81(77.14 \%)$ & $56(53.33 \%)$ & \\
\hline Positive & 73 & $24(22.86 \%)$ & $49(46.67 \%)$ & \\
\hline Number of tumours & & & & 0.831 \\
\hline Single & 185 & $92(87.62 \%)$ & $93(88.57 \%)$ & \\
\hline Multi & 25 & $13(12.38 \%)$ & $12(11.43 \%)$ & \\
\hline Tumour encapsulation & & & & 0.381 \\
\hline No & 89 & $49(46.67 \%)$ & $40(38.10 \%)$ & \\
\hline Incomplete & 57 & $28(26.67 \%)$ & $29(27.62 \%)$ & \\
\hline Full & 64 & $28(26.67 \%)$ & $36(34.29 \%)$ & \\
\hline MVI & & & & 0.024 \\
\hline Negative & 126 & $71(67.62 \%)$ & $55(52.38 \%)$ & \\
\hline Positive & 84 & $34(32.38 \%)$ & $50(47.62 \%)$ & \\
\hline
\end{tabular}

Fig. 1. PRMT1 expression was increased in HCC tissues and correlated with HCC patient survival. (A) PRMT1 mRNA levels in 35 pairs of tumour samples (T) and matched normal hepatic tissues (N) were determined by q-PCR. (B) PRMT1 protein expression levels in 10 pairs of $\mathrm{N}$ and $\mathrm{T}$ tissues and 5 pairs of $\mathrm{N}, \mathrm{T}$ and PVTT tissues (P) from HCC patients were determined by Western blot. (C) Immunohistochemistry staining of PRMT1 in paired $\mathrm{N}$ and $\mathrm{T}$ tissues from two patients. (D) Scatter plots reflecting the PRMT1 staining intensity in N $(\mathrm{n}=210)$ and $\mathrm{T}(\mathrm{n}=210)$ tissues, ${ }^{* * *}, \mathrm{P}<0.001$. (E) High PRMT1 expression correlated with worse recurrence-free survival in HCC patients (PRMT1 high, $n=105$; PRMT1 low, $n=105$ ), $P=0.010$. (F) High PRMT1 expression correlated with worse overall survival in HCC patients (PRMT1 high, $\mathrm{n}=105$; PRMT1 low, $\mathrm{n}=105$ ), $\mathrm{P}=0.001$.
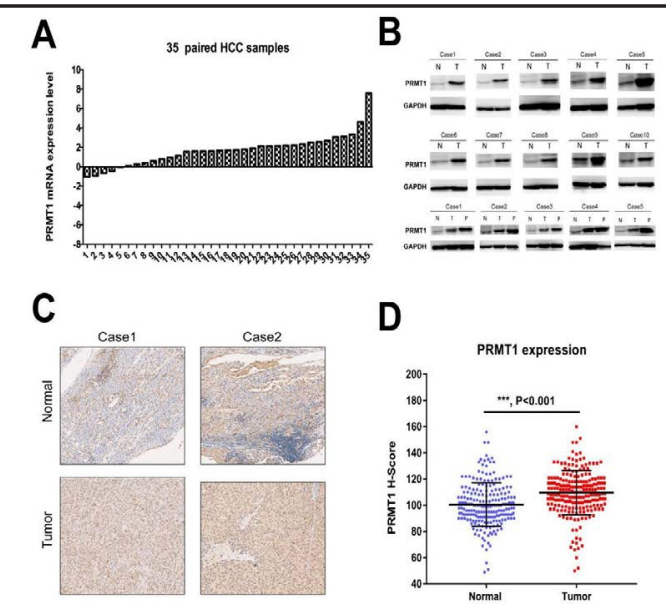

D

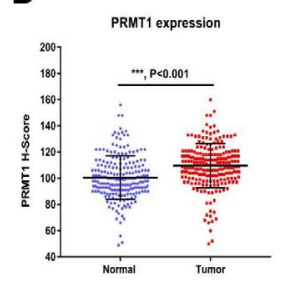

$E$

$\mathbf{F}$

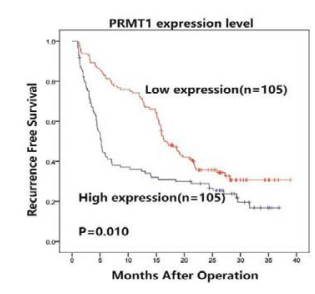

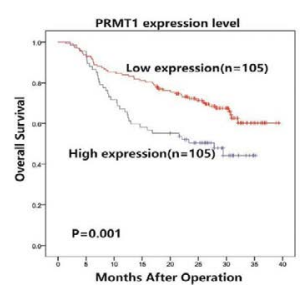


groups: PRMT1 low (score<110) and PRMT1 high (score $\geq 110$ ). We explored the relationship between PRMT1 expression and clinicopathological features in 210 HCC patients. As shown in Table 2, PRMT1 expression positivity correlated with the histologic presence of microvascular invasion $(\mathrm{P}=0.024)$, tumour differentiation $(\mathrm{P}=0.014)$, tumour size $(\mathrm{P}=$ $0.002)$, portal vein tumour thrombus (PVTT) $(\mathrm{P}=0.028)$ and small lesions $(\mathrm{P}<0.001)$. Patients with high PRMT1 expression showed a significantly poorer prognosis $(\mathrm{P}=0.001)$ and higher recurrence $(\mathrm{P}=0.010)$ than patients with low PRMT1 expression (Fig. 1E, 1F). Cox's multivariate proportional hazards model indicated that some factors were independent predictors of overall survival: PRMT1 $>110$ (hazard ratio (HR): $2.079,95 \%$ confidence interval (CI): $1.291-3.346, \mathrm{P}=0.003$ ); tumour size $>5 \mathrm{~cm}$ (HR: 2.452, 95\% CI: 1.287-4.673, P $=0.006$ ); incomplete encapsulation (HR: 0.522, 95\% CI: 0.281-0.966, P $=0.039$ ) and positive PVTT (HR: 2.413, 95\% CI: 1.307-4.45, P =0.005). The following factors were independent predictors of recurrence: PRMT1 >110 (HR: 1.552, 95\% CI: 1.160-2.354, P <=0.025); tumour size $>5 \mathrm{~cm}$ (HR: 1.548, 95\% CI: 1.034-2.318, $\mathrm{P}=0.034$ ); incomplete encapsulation (HR: 1.713, 95\% CI: 1.127-2.603, P =0.012); positive PVTT (HR: 1.830, 95\% CI: 1.107-3.027, P =0.019) and positive micro metastasis (HR: 1.501, 95\% CI: 1.034-2.179, $\mathrm{P}=0.033$ ) in HCC patients after curative resection (Tables 3 and 4). These results indicated that PRMT1 may be a tumour promoter in HCC growth and migration.

PRMT1 overexpression promoted the growth and migration of $\mathrm{HCC}$ cells

Based on the clinical data, we hypothesized that PRMT1 might influence the growth and metastasis of HCC cells. First, we compared the PRMT1 levels in the normal liver cell line LO2 and many HCC cell lines. Western blotting showed that PRMT1 expression was apparently higher in the HCC cell lines (7721, HepG2, BEL7404 and YY-8103) than in the normal cells (LO2) (Fig. 2A). Furthermore, we transfected the BEL-7404 and YY-8103 HCC cell lines with plasmids containing either an empty p23 vector or PRMT1 overexpression vectors (Flag-PRMT1) to study the biological function of PRMT1. PRMT1 expression was determined by Western blot (Fig. 2B). PRMT1 overexpression remarkably promoted the growth and colony formation of bel-7404 and

Table 3. Univariate and multivariate analyses associated with diseasefree survival. NS: No significance

\begin{tabular}{lccccc}
\hline Variables & HR $(95 \% \mathrm{CI})$ & P-value & HR & Multivariate \\
& $0.852(0.491-1.478)$ & 0.569 & & & P-value \\
\hline Male sex & $0.784(0.567-1.083)$ & 0.140 & & & \\
Age $\geq 50 \mathrm{yrs}$ & $2.164(1.478-3.167)$ & $<0.001$ & 1.974 & $1.326-2.939$ & 0.001 \\
AFP $\geq 20 \mu \mathrm{g} / \mathrm{L}$ & $1.048(0.580-1.894)$ & 0.877 & & & \\
HBsAg, Positive & $0.907(0.477-1.726)$ & 0.766 & & & \\
Ascites, Positive & $0.340(0.221-0.523)$ & $<0.001$ & 1.830 & $1.107-3.027$ & 0.019 \\
PVTT, Positive & $2.314(1.589-3.370)$ & $<0.001$ & 1.548 & $1.034-2.318$ & 0.034 \\
Tumour size $>5$ cm & $1.057(0.645-1.731)$ & 0.827 & & & - \\
No. of tumours, Multi vs single & & & & & \\
Micro metastasis, Positive & $2.405(1.729-3.347)$ & $<0.001$ & 1.501 & $1.034-2.179$ & 0.033 \\
Encapsulation, Full & & & & & \\
Encapsulation, Incomplete & $1.899(1.267-2.846)$ & 0.002 & 1.713 & $1.127-2.603$ & 0.012 \\
Encapsulation, No & $2.554(1.729-3.775)$ & $<0.001$ & 1.816 & $1.159-2.846$ & 0.009 \\
MVI, Positive & $2.313(1.669-3.204)$ & $<0.001$ & & & - \\
PRMT1 $>110$ vs $\leq 110$ & $1.727(1.193-2.380)$ & 0.010 & 1.552 & $1.160-2.354$ & 0.025 \\
\hline
\end{tabular}

Table 4. Univariate and multivariate analyses associated with overall survival. NS: No significance

\begin{tabular}{lccccc}
\hline Variables & Univariate & & Multivariate \\
& HR $(95 \% \mathrm{CI})$ & P-value & HR & $95 \%$ CI & P-value \\
\hline Male sex & $0.831(0.383-1.803)$ & 0.640 & & & \\
Age $\geq 50 \mathrm{yrs}$ & $0.877(0.569-1.353)$ & 0.554 & & & \\
AFP $\geq 20 \mu \mathrm{g} / \mathrm{L}$ & $3.040(1.681-5.501)$ & $<0.001$ & 2.706 & $1.489-4.916$ & 0.001 \\
HBsAg, Positive & $2.434(0.769-7.709)$ & 0.130 & & & \\
Ascites, Positive & $1.471(0.709-3.050)$ & 0.300 & & & \\
PVTT, Positive & $4.106(2.503-6.738)$ & $<0.001$ & 2.413 & $1.307-4.45$ & 0.005 \\
Tumour size $>5$ cm & $3.723(2.015-6.879)$ & $<0.001$ & 2.452 & $1.287-4.673$ & 0.006 \\
No. of tumours, Multi vs single & $1.182(0.627-2.230)$ & 0.605 & & & \\
Micro metastasis, Positive & $2.554(1.655-3.941)$ & $<0.001$ & & & - \\
Encapsulation, Full & & & & & \\
Encapsulation, Incomplete & $2.206(1.249-3.895)$ & 0.006 & 0.522 & $0.281-0.966$ & 0.039 \\
Encapsulation, No & $3.030(1.770-5.187)$ & 0.000 & 1.116 & $0.608-2.051$ & 0.722 \\
MVI, Positive & $2.127(1.380-3.279)$ & $<0.001$ & & & - \\
PRMT1 $>110$ vs $\leq 110$ & $2.027(1.351-3.780)$ & 0.001 & 2.079 & $1.291-3.346$ & 0.003 \\
\hline
\end{tabular}


YY-8103 HCC cells according to MTT $(\mathrm{P}<0.01)$ (Fig. 2C) and crystal violet $(\mathrm{P}<0.01)$ assays (Fig. 2D); the migration of these HCC cells was also significantly increased ( $\mathrm{P}<0.01)$ (Fig. 2E). These in vitro results showed that the overexpression of PRMT1 can benefit HCC cells.

Knocking down endogenous PRMT1 expression inhibited the growth and migration of HCC cells

To further evaluate the effects of PRMT1 in HCC cells, we knocked down the basal expression of PRMT1 in the BEL-7404 and YY-8103 HCC cell lines by RNAi (Fig. 3A). Similar to the overexpression experiments, we tested the growth, colony formation and migration of the control and RNAi cell lines. MTT assays showed a significant inhibition of proliferation

Fig. 2. PRMT1 overexpression promoted growth, colony formation and migration in HCC cells in vitro. (A) Western blots showing PRMT1 expression in five HCC cell lines: LO2, 7721, HepG2, Bel-7404 and YY-8103. GAPDH was used as a loading control. (B) Western blots showing the overexpression of PRMT1 in Bel-7404 and YY-8103 cells. (C) The effects of PRMT1 overexpression on the viability of Bel-7404 and YY-8103 cells were assessed by MTT assays. (D) The effects of PRMT1 overexpression on the viability of Bel-7404 and YY-8103 cells were assessed by crystal violet assays. (E) The effects of PRMT1 overexpression on the migration of Bel7404 and YY-8103 cells were examined by Boyden chamber assays. The data are presented as the mean \pm SEM. * ${ }^{*}<0.05$; ** $\mathrm{P}<0.01$; ${ }^{* *}, \mathrm{P}<0.001$.

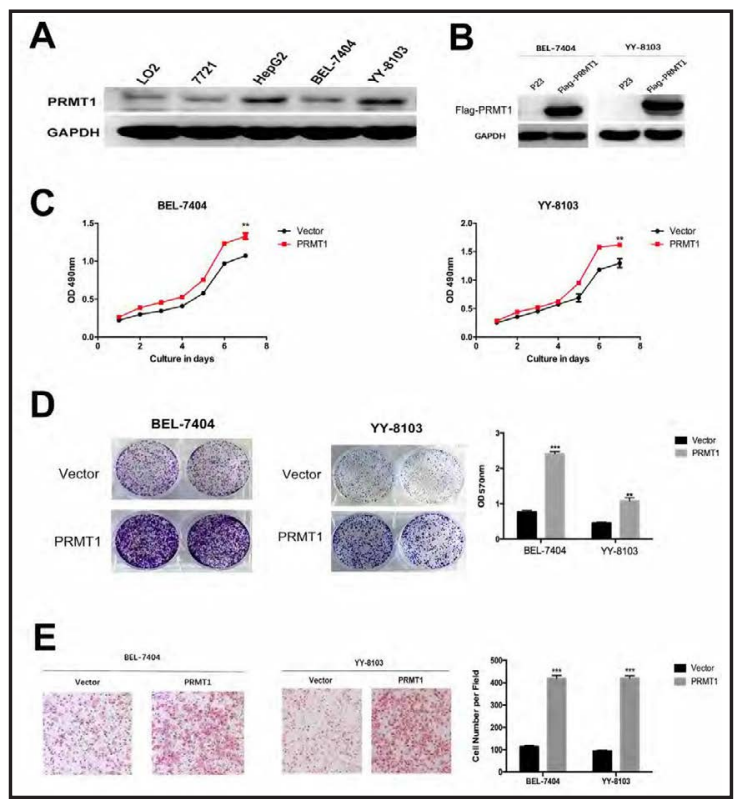

Fig. 3. PRMT1 knockdown suppressed growth, colony formation and migration in HCC cells in vitro. (A) Western blots showing the overexpression of PRMT1 in Bel-7404 and YY-8103 cells. (B) The effects of low PRMT1 expression on the viability of Bel-7404 and YY-8103 cells were assessed by MTT assays. (C) The effects of PRMT1 knockdown on the viability of Bel-7404 and YY-8103 cells were assessed by crystal violet assays. (D) The effects of low PRMT1 expression on the migration of Bel7404 and YY-8103 cells were examined by Boyden chamber assays. The data are presented as the mean \pm SEM. * $\mathrm{P}<0.05$; ** $\mathrm{P}<0.01$; ***, $\mathrm{P}<0.001$.

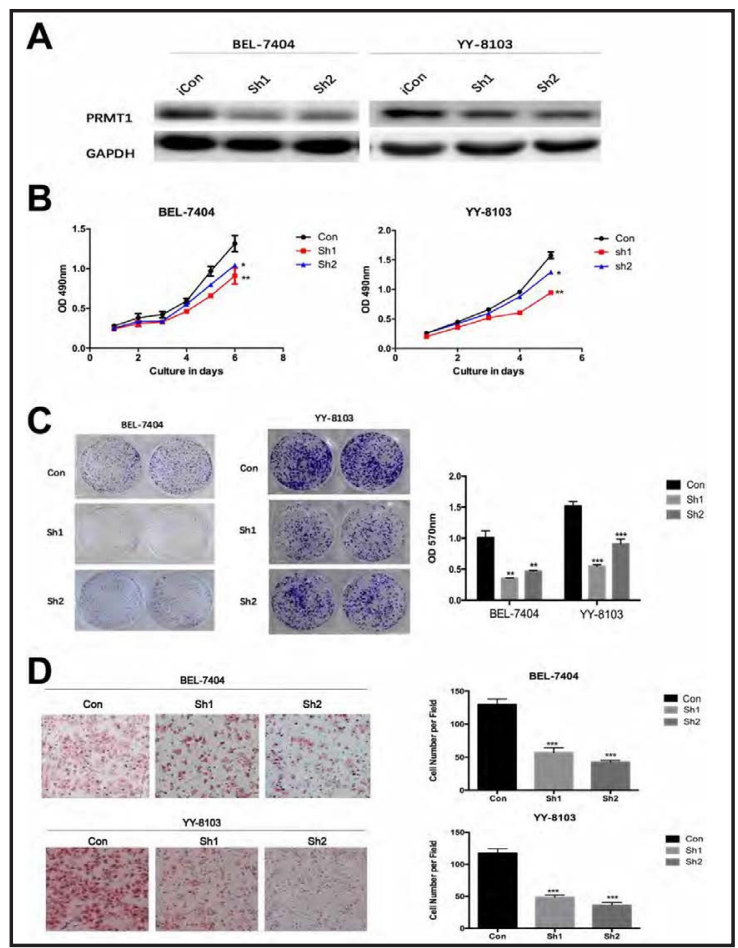


after the downregulation of PRMT1 expression in the BEL-7404 and YY-8103 HCC cell lines $(\mathrm{P}<0.05)$ (Fig. 3B). Colony formation assays showed a significant reduction in the number of colonies formed after PRMT1 downregulation in these HCC cell lines compared with that in the control cell lines ( $\mathrm{P}<0.01$ ) (Fig. 3C). Boyden chamber assays indicated significantly reduced migration in PRMT1 RNAi HCC cells (Fig. 3D). These results demonstrated that knocking down PRMT1 might play a protective role in HCC by decreasing the growth and migration of HCC cells.

Knocking down PRMT1 suppressed tumourigenesis in HCC cells in vivo

Based on the in vitro experiments, control and PRMT1 knockdown YY-8103 cells were injected subcutaneously into the flanks of nude mice, and tumour growth was monitored (Fig. 4B). The tumours generated by YY-8103 cells with PRMT1 knockdown grew slowly $(\mathrm{P}<0.001)$ and were smaller and lighter than those generated by the control cells $(n=5$, $\mathrm{P}=0.0274$ ) (Fig. 4A-4C). These results indicated that PRMT1 may be an oncogene that promotes tumourigenesis in HCC cells in vivo.

PRMT1 enhanced the intrahepatic metastatic potential of HCC cell lines in vivo

From the preliminary in vitro study, we hypothesized that PRMT1 might promote the metastasis of HCC cells in vivo. We established control and PRMT1 knockdown YY-8103 cells that overexpressed an exogenous luciferase gene. These two cell lines were injected intraperitoneally into left liver lobes of nude mice. The metastasis of YY-8103 cells in the livers was monitored by an in vivo imaging system. As shown in Fig. 4D and 4E, knocking down PRMT1 expression inhibited the intrahepatic metastasis of YY-8103 cells $(\mathrm{P}<0.001)$. These results revealed that knocking down PRMT1 inhibited the metastasis of HCC cells in vivo. These in vivo experiments agreed with the experiments showing that PRMT1 promoted the growth of HCC cells in vitro.

\section{PRMT1 activated STAT3 signalling in HCC cells}

It has been reported that constitutive JAK/STAT3 signalling pathway activation plays an important role in the development and progression of hepatocellular carcinoma (HCC). To find the underlying mechanism of PRMT1 in HCC, we measured STAT3 phosphorylation and total STAT3 protein levels in PRMT1 overexpression and knockdown cell lines. Western blotting showed that PRMT1 increased the levels of STAT3 phosphorylation (Fig. 5A). Then,

Fig. 4. PRMT1 overexpression promoted the tumourigenesis and intrahepatic metastasis of HCC cells in vivo. (A) Representative images of the tumours generated by controls and PRMT1 knockdown YY-8103 cells. (B) Growth curves of the tumours $\left(\mathrm{cm}^{3}\right)$. (C) Weights of the tumours (g). (D) Monitoring of the intrahepatic metastasis of control and PRMT1 knockdown YY-8103 cells through day 30 . The images were obtained at the indicated time points after injection. (E) The mean photon counts of each group of mice were quantified and are shown for day 30. Each bar represents the mean \pm SD. ${ }^{*} \mathrm{P}<0.05 ;{ }^{* *} \mathrm{P}<0.01 ;{ }^{* * *}, \mathrm{P}<0.001$.

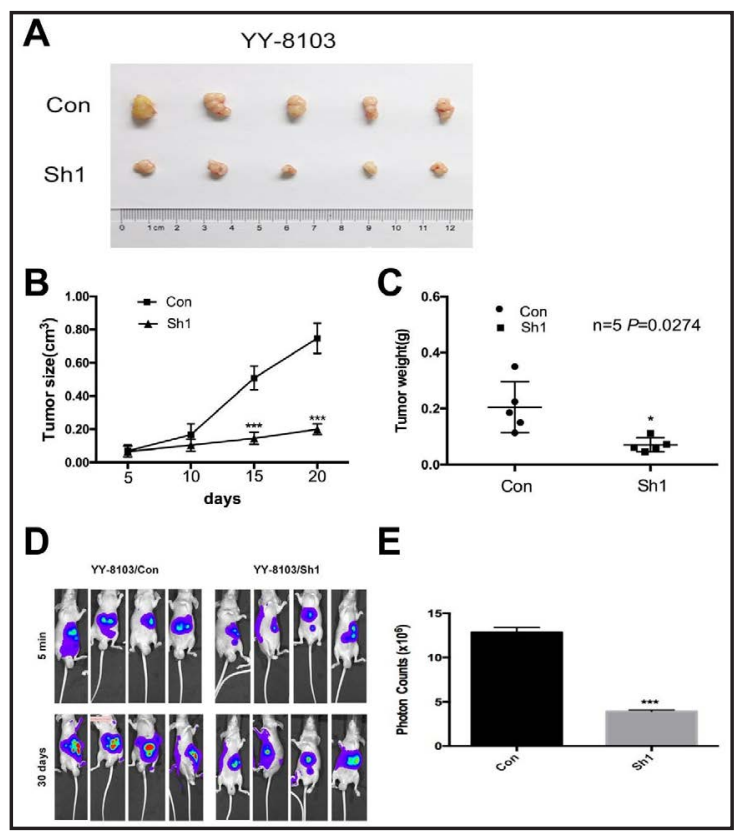




\section{Cellular Physiology Cell Physiol Biochem 2018:47:1643-1654 and Biochemistry \begin{tabular}{c|c} 
DOI: 10.1159/000490983 \\
Published online: June 27, 2018 & $\begin{array}{l}\text { O 2018 The Author(s). Published by S. Karger AG, Basel } \\
\text { www.karger.com/cpb }\end{array}$
\end{tabular} Zhang et al.: PRMT1 Promoted HCC Growth and Metastasis}

Fig. 5. PRMT1 activated STAT3 signalling in HCC cells. (A) The overexpression and knockdown of PRMT1 respectively activated and suppressed STAT3 signalling in Bel-7404 and YY-8103 cells according to Western blot. GAPDH was used as a loading control. (B) The overexpression and knockdown of PRMT1 respectively activated and suppressed STAT3 signalling in Bel-7404 and YY8103 cells according to luciferase reporter assays. (C) Changes in the molecules downstream of the STAT3 signalling pathway, namely VEGF $\alpha$, IL-6 and C-MYC, were examined by q-PCR in PRMT1 knockdown YY-8103 cells. The data are presented as the mean $\pm \mathrm{SEM}, *, \mathrm{P}<0.05$; **, $\mathrm{P}<0.01$; ***, $\mathrm{P}<0.001$.

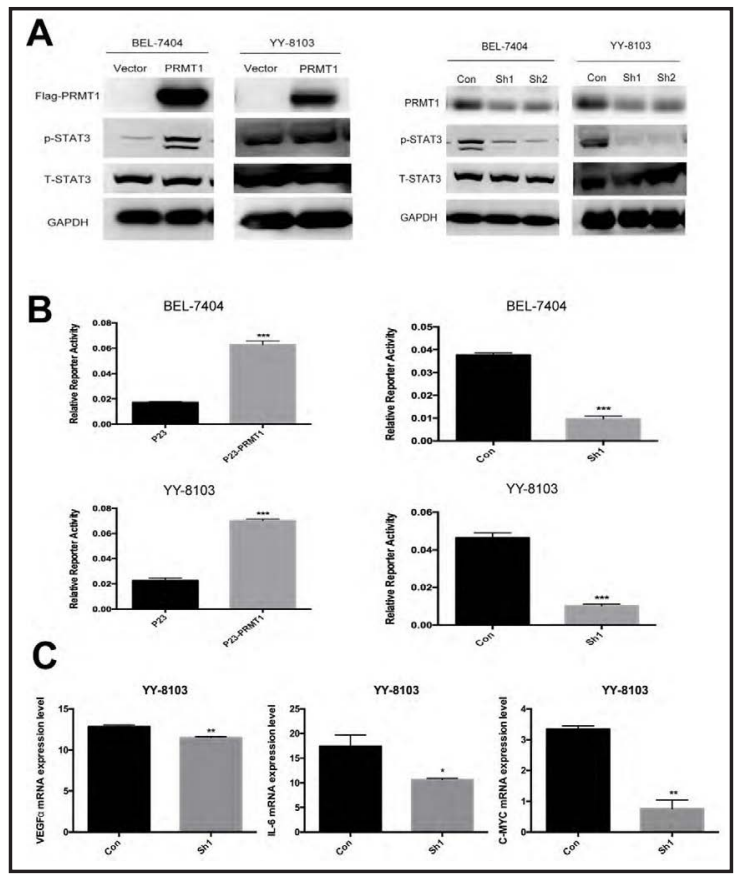

we conducted screening via a luciferase reporter assay. The results indicated that PRMT1 overexpression increased the reporter gene for STAT3 signalling, and PRMT1 downregulation inhibited STAT3 signalling (Fig. 5B). Changes in the molecules downstream of the STAT3 signalling pathway were examined by q-PCR in PRMT1 knockdown YY-8103 cells. mRNA expression levels of VEGF $\alpha$, IL-6 and C-MYC were low because PRMT1 knockdown influenced STAT3 signalling (Fig. 5C). Taken together, all these data suggest that PRMT1 can activate STAT3 phosphorylation in STAT3 signalling.

Cryptotanshinone, a potent STAT3 inhibitor, inhibited STAT3 phosphorylation and reversed the HCC phenotype of high PRMT1 expression

Cryptotanshinone is a potent STAT3 inhibitor that can inhibit STAT3 phosphorylation [14]. The concentrations and treatment duration of cryptotanshinone were $5 \mu \mathrm{M}$ in DMSO and 24 h. Cryptotanshinone was incubated with Bel-7404 and YY-8103 cells overexpressing PRMT1 to confirm whether STAT3 pathway activation could be blocked to suppress the growth and migration of HCC cells. First, cryptotanshinone inhibited STAT3 phosphorylation in the PRMT1 overexpression Bel-7404 and YY-8103 cells according to Western blots (Fig. 6A). Second, MTT assays indicated that PRMT1 overexpression remarkably promoted the growth of Bel-7404 and YY-8103 HCC cells, but cryptotanshinone treatment in these HCC cells could not (Fig. 6B). The same results were shown by colony formation assays using crystal violet staining (Fig. 6C) and by cell migration assays using Boyden chambers in these HCC cells $(\mathrm{P}<0.01)$ (Fig. 6D). The above results may show that PRMT1 plays a role in promoting HCC by activating the STAT3 pathway and blocking STAT3 phosphorylation because cryptotanshinone, a potent STAT3 inhibitor, suppressed the HCC growth and migration.

\section{Discussion}

Most previous studies have indicated that the PRMT family plays an important role in gene transcription regulation and thus regulates many biological and pathological processes, such as DNA repair, cell growth and tumourigenesis $[15,16]$. Recent reports have revealed the relationship between various cancers and PRMT1, which is overexpressed in breast 


\section{Cellular Physiology Cell Physiol Biochem 2018;47:1643-1654 \begin{tabular}{l|l} 
DOI: 10.1159/000490983 & a 2018 The Author(s). Published by S. Karger AG, Basel \\
www.karger.com/cpb
\end{tabular} Zhang et al.: PRMT1 Promoted HCC Growth and Metastasis}

Fig. 6. Cryptotanshinone, a potent STAT3 inhibitor, inhibited STAT3 phosphorylation and reversed the HCC phenotype of PRMT1 expression. (A) Cryptotanshinone inhibited STAT3 phosphorylation in Bel-7404 and YY8103 cells overexpressing PRMT1 according to Western blots. (B) The effects of inhibiting STAT3 phosphorylation on the viability of Bel7404 and YY-8103 cells were assessed by MTT assays. (C) The effects of inhibiting STAT3 phosphorylation on the viability of Bel-7404 and YY-8103 cells were assessed by crystal violet assays. (D) The effects of inhibiting STAT3 phosphorylation on the migration of Bel-7404 and YY-8103 cells were examined by Boyden chamber assays. The data are presented as the mean \pm SEM. * $\mathrm{P}<0.05$; **, $\mathrm{P}<0.01$; ***, $\mathrm{P}<0.001$.

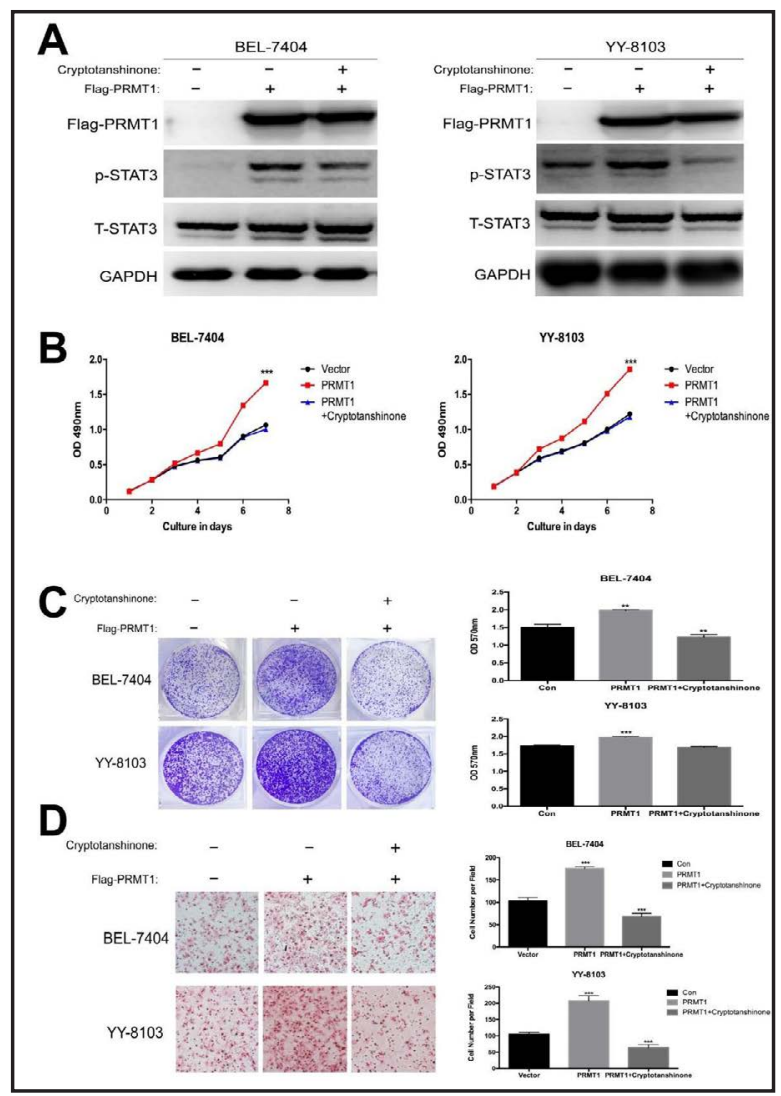

cancer, bladder cancer and lung cancer [17-20].. Notably, PRMT1 was recently reported to be upregulated through miR-503 in HCC [21], and PRMT1 may promote the proliferation and metastasis of HCC cells in vitro [13]. However, these reports did not provide enough evidence, such as in vivo experiments, to prove this role of PRMT1 and the underlying mechanism in HCC. This is the first report describing the clinical significance of positive PRMT1 expression in human HCC and its correlation with malignant behaviour, recurrence and prognosis. We revealed a significant role of PRMT1 in the tumour progression and metastasis of HCC through STAT3 signalling pathway activation.

According to the clinical data, this study shows that PRMT1 expression was higher in human HCC tissues than in paired normal tissues. HCC patients with high PRMT1 expression had worse prognoses and higher recurrence rates than those with low PRMT1 expression. Furthermore, PRMT1 overexpression, as well as large tumour size, incomplete encapsulation and positive PVTT, was an independent and significant risk factor for survival and recurrence according to the multivariate analysis. These clinical results revealed that PRMT1 may be a reliable biomarker for HCC and could be important for improving treatment strategies for HCC patients. Inhibiting the cellular signal transduction pathway activated by PRMT1 overexpression in HCC patients could be a potential treatment method.

In basic studies, we found that PRMT1 overexpression could promote HCC cell proliferation and migration in vitro. In addition, PRMT1 also promoted tumour growth and intrahepatic metastasis in vivo in mice. However, PRMT1 knockdown significantly inhibited the malignant abilities both in vitro and in vivo. In experiments investigating the underlying mechanism, PRMT1 accelerated HCC through the STAT3 pathway at the mRNA and protein levels. We also found that upregulating PRMT1 can increase STAT3 phosphorylation and thereby activate the STAT3 pathway, which can potentiate the development of HCC [22-27]. Finally, cryptotanshinone, a STAT3 inhibitor, suppressed HCC growth and migration through inhibiting STAT3 phosphorylation, which was activated by PRMT1 overexpression. 


\section{Cellular Physiology Cell Physiol Biochem 2018;47:1643-1654 \begin{tabular}{lll} 
DOI: 10.1159/000490983 & and Biochemistry & $\begin{array}{l}\text { O } 2018 \text { The Author(s). Published by S. Karger AG, Basel } \\
\text { wwww.karger.com/cpb }\end{array}$ \\
\cline { 2 - 3 }
\end{tabular} \\ Published online: June 27, 2018}

Previous studies indicated that PRMT1 overexpression led to the progression and metastasis of various cancers and predicted a worse prognosis in patients $[10,12,13]$. Furthermore, Nakai, et al. reported that EGFR methylation and activity were significantly reduced in PRMT1 knockdown cells compared to the triple-negative breast cancer parental cells [9]. PRMT1 inhibition may be an effective therapeutic strategy for overcoming intrinsic cetuximab resistance in triple-negative breast cancer. In human melanoma, Li, et al. reported that PRMT1 is overexpressed in human melanoma and may regulate tumour growth and metastasis via targeting ALCAM [8]. Eberhardt, et al. indicated that PRMT1 is a novel regulator of MYCN, which implicates PRMT1 as a potential therapeutic target in neuroblastoma pathogenesis [28]. These reports might also support the correlation between PRMT1 and HCC progression, specifically tumour growth and metastasis, which was shown in this study. What is more, a STAT3 inhibitor was shown to benefit HCC patients with PRMT1 overexpression in a clinical study.

In conclusion, we provided novel insight into the significance of PRMT1 expression in malignant behaviour, recurrence and prognosis in HCC. Our findings revealed that PRMT1 could play a crucial role in tumour progression and metastasis in HCC through activating the phosphorylation of STAT3 and the STAT3 pathway. PRMT1 could serve as a valuable biomarker and potential molecular target for the development of HCC therapies. These results are important for determining the relationship between PRMT1 and HCC and finding novel HCC biomarkers.

\section{Disclosure Statement}

The authors declare to have no competing interests.

\section{References}

1 Torre LA, Bray F, Siegel RL, Ferlay J, Lortet-Tieulent J, Jemal A: Global cancer statistics, 2012 CA: Cancer J Clinic 2015;65:87-108.

-2 Yu WB, Rao A, Vu V, Xu L, Rao JY, Wu JX: Management of centrally located hepatocellular carcinoma: Update 2016. World J Hepatol 2017;9:627-634.

3 Omata M, Cheng AL, Kokudo N, Kudo M, Lee JM, Jia J, Tateishi R, Han KH, Chawla YK, Shiina S, Jafri W, Payawal DA, Ohki T, Ogasawara S, Chen PJ, Lesmana CRA, Lesmana LA, Gani RA, Obi S, Dokmeci AK, Sarin SK: Asia-pacific clinical practice guidelines on the management of hepatocellular carcinoma: A 2017 update. Hepatol Int 2017;11:317-370.

-4 Zhang X, Wang K, Wang M, Yang G, Ye X, Wu M, Cheng S: Transarterial chemoembolization (tace) combined with sorafenib versus tace for hepatocellular carcinoma with portal vein tumor thrombus: A systematic review and meta-analysis. Oncotarget 2017;8:29416-29427.

5 Jing W, Luo P, Zhu M, Ai Q, Chai H, Tu J: Prognostic and diagnostic significance of sdpr-cavin-2 in hepatocellular carcinoma. Cell Physiol Biochem 2016;39:950-960.

-6 Portolani N, Coniglio A, Ghidoni S, Giovanelli M, Benetti A, Tiberio GA, Giulini SM: Early and late recurrence after liver resection for hepatocellular carcinoma: Prognostic and therapeutic implications. Ann Surg 2006;243:229-235.

-7 Harlan LC, Parsons HM, Wiggins CL, Stevens JL, Patt YZ: Treatment of hepatocellular carcinoma in the community: Disparities in standard therapy. Liver Cancer 2015;4:70-83.

-8 Yang F, Lv L, Zhang K, Cai Q Liu J, Jiang Y: Elevated foxc2 expression promotes invasion of hcc cell lines and is associated with poor prognosis in hepatocellular carcinoma. Cell Physiol Biochem 2017;44:99-109.

-9 Lee YH, Stallcup MR: Minireview: Protein arginine methylation of nonhistone proteins in transcriptional regulation. Mol Endocrinol 2009;23:425-433.

10 Li L, Zhang Z, Ma T, Huo R: Prmt1 regulates tumor growth and metastasis of human melanoma via targeting alcam. Mol Med Report 2016;14:521-528. 


\section{Cellular Physiology Cell Physiol Biochem 2018;47:1643-1654

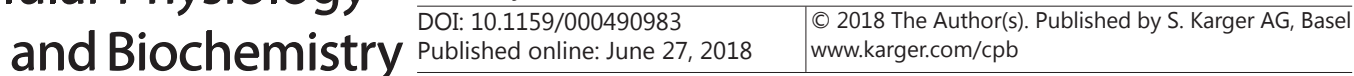

11 Nakai K, Xia W, Liao HW, Saito M, Hung MC, Yamaguchi H: The role of prmt1 in egfr methylation and signaling in mda-mb-468 triple-negative breast cancer cells. Breast Cancer 2018;25:74-80.

12 Zhou W, Yue H, Li C, Chen H, Yuan Y: Protein arginine methyltransferase 1 promoted the growth and migration of cancer cells in esophageal squamous cell carcinoma. Tumour Biol 2016;37:2613-2619.

13 Gou Q He S, Zhou Z: Protein arginine n-methyltransferase 1 promotes the proliferation and metastasis of hepatocellular carcinoma cells. Tumour Biol 2017;39:1010428317691419.

14 Shin DS, Kim HN, Shin KD, Yoon YJ, Kim SJ, Han DC, Kwon BM: Cryptotanshinone inhibits constitutive signal transducer and activator of transcription 3 function through blocking the dimerization in du145 prostate cancer cells. Cancer Res 2009;69:193-202.

-15 Takahashi Y, Daitoku H, Yokoyama A, Nakayama K, Kim JD, Fukamizu A: The c. Elegans prmt-3 possesses a type iii protein arginine methyltransferase activity. J Recept Signal Transduct Res 2011;31:168-172.

16 Ahmad A, Dong Y, Cao X: Characterization of the prmt gene family in rice reveals conservation of arginine methylation. PLoS One 2011;6:e22664.

17 Chuang CY, Chang CP, Lee YJ, Lin WL, Chang WW, Wu JS, Cheng YW, Lee H, Li C: Prmt1 expression is elevated in head and neck cancer and inhibition of protein arginine methylation by adenosine dialdehyde or prmt1 knockdown downregulates proliferation and migration of oral cancer cells. Oncol Rep 2017;38:1115-1123.

18 Hsu JH, Hubbell-Engler B, Adelmant G, Huang J, Joyce CE, Vazquez F, Weir BA, Montgomery P, Tsherniak A, Giacomelli AO, Perry JA, Trowbridge J, Fujiwara Y, Cowley GS, Xie H, Kim W, Novina CD, Hahn WC, Marto JA, Orkin SH: Prmt1-mediated translation regulation is a crucial vulnerability of cancer. Cancer Res 2017;77:4613-4625.

19 Montenegro MF, Gonzalez-Guerrero R, Sanchez-del-Campo L, Pinero-Madrona A, Cabezas-Herrera J, Rodriguez-Lopez JN: Targeting the epigenetics of the DNA damage response in breast cancer. Cell Death Dis 2016;7:e2180.

20 Zhang T, Cui G, Yao YL, Guo Y, Wang QC, Li XN, Feng WM: Inhibition of nonsmall cell lung cancer cell migration by protein arginine methyltransferase 1-small hairpin rna through inhibiting epithelialmesenchymal transition, extracellular matrix degradation, and src phosphorylation in vitro. Chin Med J (Engl) 2015;128:1202-1208.

21 Li B, Liu L, Li X, Wu L: Mir-503 suppresses metastasis of hepatocellular carcinoma cell by targeting prmt1 Biochem Biophys Res Commun 2015;464:982-987.

22 Wang H, Huo X, Yang XR, He J, Cheng L, Wang N, Deng X, Jin H, Wang N, Wang C, Zhao F, Fang J, Yao M, Fan J, Qin W: Stat3-mediated upregulation of Incrna hoxd-as1 as a cerna facilitates liver cancer metastasis by regulating sox4. Mol Cancer 2017;16:136.

23 Sakurai T, Yada N, Hagiwara S, Arizumi T, Minaga K, Kamata K, Takenaka M, Minami Y, Watanabe T, Nishida $\mathrm{N}$, Kudo M: Gankyrin induces stat3 activation in tumour microenvironment and sorafenib resistance in hepatocellular carcinoma. Cancer Sci 2017;108:1996-2003

24 Gao Y, Li W, Liu R, Guo Q Li J, Bao Y, Zheng H, Jiang S, Hua B: Norcantharidin inhibits il-6-induced epithelialmesenchymal transition via the jak2/stat3/twist signaling pathway in hepatocellular carcinoma cells. Oncol Rep 2017;38:1224-1232.

25 Gao L, Wang L, Sun Z, Li H, Wang Q, Yi C, Wang X: Morusin shows potent antitumor activity for human hepatocellular carcinoma in vitro and in vivo through apoptosis induction and angiogenesis inhibition. Drug Des Devel Ther 2017;11:1789-1802.

-26 Liu CY, Chen KF, Chao TI, Chu PY, Huang CT, Huang TT, Yang HP, Wang WL, Lee CH, Lau KY, Tsai WC, Su JC, Wu CY, Chen MH, Shiau CW, Tseng LM: Sequential combination of docetaxel with a shp-1 agonist enhanced su ppression of p-stat3 signaling and apoptosis in triple negative breast cancer cells. J Mol Med (Berl) 2017;95:965-975.

27 Mao J, Hu X, Pang P, Zhou B, Li D, Shan H: Mir-30e acts as a tumor suppressor in hepatocellular carcinoma partly via jak1/stat3 pathway. Oncol Rep 2017;38:393-401.

28 Eberhardt A, Hansen JN, Koster J, Lotta LT, Jr., Wang S, Livingstone E, Qian K, Valentijn LJ, Zheng YG, Schor NF, Li X: Protein arginine methyltransferase 1 is a novel regulator of mycn in neuroblastoma. Oncotarget 2016;7:63629-63639. 